\title{
Vortices in U(1) noncommutative gauge fields
}

\author{
H. Falomir* \\ IFLP-Departamento de Física, Facultad de Ciencias Exactas, Universidad Nacional de la Plata, C.C. 67, (1900) La Plata, Argentina \\ J. Gamboa, ${ }^{\dagger}$ J. López-Sarrión, ${ }^{\dagger}$ and F. Méndez ${ }^{\S}$ \\ Departamento de Física, Universidad de Santiago de Chile, Casilla 307, Santiago 2, Chile \\ A. J. da Silvall \\ Instituto de Física, Universidade de São Paulo, CP 66316, 05315-970, São Paulo, SP, Brazil \\ (Received 28 June 2006; published 25 August 2006)
}

\begin{abstract}
Charged vortex solutions for the noncommutative Maxwell-Higgs model in $3+1$ dimensions are found. We show that the stability of these vortex solutions is lost for some, large enough, noncommutativity parameters. A nontopological charge, however, is induced by noncommutative effects.
\end{abstract}

DOI: 10.1103/PhysRevD.74.047701

PACS numbers: 11.15.Tk, 11.10.Nx

\section{INTRODUCTION}

Lorentz and CPT symmetries are cornerstones in relativistic quantum field theory which are casting doubt in connection with recent experiments in cosmic physics rays [1], neutrino physics [2], and cosmological measurements [3].

In order to explain these experimental results, some authors have suggested conservative avenues; however, explaining experiments like LNSD and some other astrophysical observations apparently will require new and radical ideas.

On the other hand, vortices are localized, classical, finite energy solutions that appear in some quantum field theory models $[4,5]$. Such solutions encode the collective behavior of some systems at a nonperturbative level. Although these kinds of solutions originally were found in condensed matter physics [6], they also play an important role in particle physics [7] and cosmology, in the language of cosmic strings [8] and superstrings [9].

A particular model that exhibits this solution is the Maxwell-Higgs model in $3+1$ dimensions. It has also been shown [5] that properties of vortices are modified if we add to the theory a Chern-Simons (CS) term. For instance, they acquire a finite quantized charge and angular momentum.

On the other hand, in previous papers [10,11], and from a completely different point of view, we have reported that a particular Lorentz invariance violation (LIV) induces a CS term in Abelian and non-Abelian Maxwell theories. Our approach to LIV contains two scales, infrared and ultraviolet, and it is properly defined in the field space rather than in spacetime.

\footnotetext{
*Electronic address: falomir@fisica.unlp.edu.ar

†Electronic address: jgamboa@lauca.usach.cl

${ }^{\ddagger}$ Electronic address: justo@dftuz.unizar.es

${ }^{\S}$ Electronic address: fmendez@lauca.usach.cl

"Electronic address: ajsilva@fma.if.usp.br
}

Therefore, it is natural to ask if our approach admits vortexlike solutions and, in this case, which are their properties.

In this paper we discuss some new features arising when an infrared cutoff is introduced into the Maxwell-Higgs model in $3+1$ dimensions, describing the way the uncharged vortex solutions for the standard Maxwell-Higgs model are modified and considering the stability of these solutions.

As discussed in [10-13] (see also [14]), an explicit infrared cutoff appears in quantum field theory when the canonical commutators of momenta are modified in analogy with the Landau problem in quantum mechanics. The presence of this cutoff breaks Lorentz symmetry and induces a kind of dimensional reduction which could provide a new clue for many unsolved problems in quantum field theory, cosmology, and astrophysics.

Let us start reviewing the main aspects of quantum theory with noncommutative fields. Later, we will show that a theory with noncommutative $\mathrm{U}(1)$ fields coupled to a Higgs scalar has indeed vortex solutions.

In Refs. [10,11,13], an approach to a Lorentz invariance violating quantum field theory has been proposed, inspired by noncommutative geometry. The fields, instead of satisfying the standard canonical commutators, obey

$$
\begin{aligned}
& {\left[\phi_{i}(\vec{x}), \phi_{j}(\vec{y})\right]=i \theta_{i j} \delta(\vec{x}-\vec{y}),} \\
& {\left[\pi_{i}(\vec{x}), \pi_{j}(\vec{y})\right]=i \mathcal{B}_{i j} \delta(\vec{x}-\vec{y}),} \\
& {\left[\phi_{i}(\vec{x}), \pi_{j}(\vec{y})\right]=i \delta_{i j} \delta(\vec{x}-\vec{y}),}
\end{aligned}
$$

where $i, j, \ldots=1,2,3, \ldots$ are internal indices and $\theta_{i j}$ and $\mathcal{B}_{i j}$ are scales with dimensions of (energy) ${ }^{-1}$ and energy, respectively. For small values of these scales, they correspond to weak ultraviolet and infrared Lorentz invariance violations, respectively.

At this point, it is worth noting that this approach does not correspond to the noncommutative geometry in the 
usual sense, where one assumes the commutator

$$
[x, y] \sim \theta .
$$

Rather, while the commutators (1) violate the microcausality principle imposing an ultraviolet scale, (2) affects the physics in the infrared sector of the quantum field theory [15].

The noncommutative scalar electrodynamics is defined in full analogy with the electrodynamics, but modifying appropriately the canonical commutators.

The noncommutative scalar electrodynamics we will consider in the present article is defined in full analogy with the electrodynamics, but modifying appropriately the canonical commutators. (For a model based on the noncommutativity of coordinates and the Moyal product for functions, see [16])

The Hamiltonian for this model becomes

$$
\begin{aligned}
\mathcal{H}= & \int d^{3} x\left\{\frac{1}{2}\left(E^{2}+B^{2}\right)-A^{0}(\vec{\nabla} \cdot \vec{E}+\vec{\theta} \cdot \vec{B})\right. \\
& +\left(\Pi^{*}+i e A_{0} \phi\right)\left(\Pi-i e A_{0} \phi^{*}\right)-e^{2} A_{0}^{2} \phi^{*} \phi \\
& \left.+e \Theta \phi^{*} \phi A^{0}+(\vec{D} \phi)^{*} \cdot(\vec{D} \phi)+V\left(\phi^{*} \phi-\phi_{0}^{*} \phi_{0}\right)\right\},
\end{aligned}
$$

where $V\left(\phi^{*} \phi-\rho_{0}\right)$ is a scalar potential-responsible for the symmetry breaking — which can be written as

$$
V\left(\phi^{*} \phi-\phi_{0}^{*} \phi_{0}\right)=\frac{\lambda}{4}\left(\phi^{*} \phi-\phi_{0}^{*} \phi_{0}\right)^{2},
$$

with $\lambda$ and $\phi_{0}$ real and complex constants, respectively.

The modified commutators we adopt are

$$
\begin{aligned}
{\left[E_{i}(\vec{x}), E_{j}(\vec{y})\right] } & =i \epsilon_{i j k} \theta^{k} \delta^{3}(\vec{x}-\vec{y}), \\
{\left[\Pi^{*}(\vec{x}), \Pi(\vec{y})\right] } & =\Theta \delta^{3}(\vec{x}-\vec{y}),
\end{aligned}
$$

where $E_{i}$ is the electric field and $\Pi$ is the canonical momentum associated to the charged field $\phi$. Note that the Gauss law is modified as in (4), in order to keep gauge invariance with the modified commutators (5a) and (5b).

\section{EQUATION OF MOTION AND VORTEX SOLUTION}

In order to write explicitly the equations of motion (e.o.m.) for this system, we will take a coordinate system in which the third axis $(z)$ is along the direction of the (spacelike) vector $\vec{\theta}$, that is, $\vec{\theta}=(0,0, \theta)$. Then, the e.o.m. for the Hamiltonian (4) with Poisson brackets structure (5) become

$$
\begin{aligned}
\partial_{\mu} F^{\mu \nu}+\frac{\theta}{2} \epsilon^{3 \nu \alpha \beta} F_{\alpha \beta} & =J^{\nu}-\delta_{0}^{\nu} e \Theta \phi^{*} \phi, \\
D^{\mu} D_{\mu} \phi+\frac{\partial V}{\partial \rho} \phi+i \Theta D^{0} \phi & =0,
\end{aligned}
$$

where

$$
\begin{aligned}
J^{\mu} & :=-i e\left[\phi^{*}\left(D^{\mu} \phi\right)-\left(D^{\mu} \phi\right)^{*} \phi\right] \\
& =-i e\left[\phi^{*} \partial^{\mu} \phi-\phi \partial^{\mu} \phi^{*}\right]-2 e^{2} A^{\mu} \phi^{*} \phi,
\end{aligned}
$$

and

$D_{\mu} \phi=\partial_{\mu} \phi-i e A_{\mu} \phi, \quad\left(D_{\mu} \phi\right)^{*}=\partial_{\mu} \phi^{*}+i e A_{\mu} \phi^{*}$,

with $\rho:=\phi^{*} \phi$.

Using the Coulomb gauge condition, $\vec{\nabla} \cdot \vec{A}=\partial_{i} A_{i}=0$, and considering static and $z$ coordinate independent configurations, one obtains the equations

$$
\begin{gathered}
-\nabla^{2} A^{0}+\theta B_{\theta}=-e\left(2 e A^{0}-\Theta\right) \phi^{*} \phi \\
-\nabla^{2} A^{i}-\theta \epsilon^{i j} F_{0 j}=J^{i}, \quad i, j=1,2, \\
-(\vec{D})^{2} \phi+\frac{\partial V}{\partial \rho} \phi-e A^{0}\left(e A^{0}-\Theta\right) \phi=0 \\
-\nabla^{2} A^{3}=-2 e^{2} A^{3} \phi^{*} \phi
\end{gathered}
$$

which can be simplified by calling

$$
\begin{aligned}
& \tilde{A}^{0}=A^{0}-\frac{\Theta}{2 e} \\
& \tilde{\phi}_{0}=e^{i \zeta} \sqrt{\rho_{0}-\frac{\Theta^{2}}{2 \lambda}} \\
& \tilde{\phi}_{0}^{*}=e^{-i \zeta} \sqrt{\rho_{0}-\frac{\Theta^{2}}{2 \lambda}}
\end{aligned}
$$

and redefining the scalar potential as

$$
\tilde{V}(\rho)=V\left(\rho-\rho_{0}\right)+\frac{\Theta^{2}}{4} \rho .
$$

In such a way, the set of equations for the static noncommutative scalar electrodynamics turns out to be

$$
\begin{gathered}
-\nabla^{2} \tilde{A}_{0}-\frac{\theta}{2} \epsilon_{i j} F_{i j}=-2 e^{2} \tilde{A}_{0} \phi^{*} \phi, \\
-\nabla^{2} A_{i}+\theta \epsilon_{i j} \partial_{j} \tilde{A}_{0}=J_{i}, \\
-(\vec{D})^{2} \phi+\frac{\lambda}{2}\left(\phi^{*} \phi-\tilde{\phi}_{0}^{*} \tilde{\phi}_{0}\right) \phi-\left(e \tilde{A}_{0}\right)^{2} \phi=0,
\end{gathered}
$$

which are just the equations for the static Maxwell-ChernSimons-Higgs model [17].

But, contrary to the commutative electrodynamics case, the linear charge density operator is here shifted according to

$$
Q_{\theta}=\int d^{2} x J^{0}(x)=\tilde{Q}_{\theta}-e \Theta \int d^{2} x \phi^{*} \phi,
$$

where

$$
\begin{aligned}
\tilde{Q}_{\theta} & \equiv \int d^{2} x \tilde{J}^{0} \\
& =\int d^{2} x\left\{-i e\left(\phi^{*} \partial^{0} \phi-\phi \partial^{0} \phi^{*}\right)-2 e^{2} \tilde{A}^{0}\right\} .
\end{aligned}
$$


In order to analyze the asymptotic behavior of the fields we choose cylindrical coordinates $(r, \varphi, z)$ and follow Ref. [5]. Thus, for $r \rightarrow \infty$ we have

$$
\begin{aligned}
\phi & \rightarrow \tilde{\phi}_{0} e^{i \alpha(\varphi)}+\mathcal{O}\left(e^{-\lambda r}\right) \\
A_{i} & \rightarrow \frac{1}{e} \partial_{i} \alpha(\varphi)+\mathcal{O}\left(e^{-r / r_{0}}\right) \\
\tilde{A}_{0} & \rightarrow \frac{1}{e} \partial_{0} \alpha(\varphi)+\mathcal{O}\left(e^{-r / r_{0}}\right)=0+\mathcal{O}\left(e^{-r / r_{0}}\right),
\end{aligned}
$$

with $r_{0}$ a constant with appropriate dimensions. What is important here is the exponential behavior of the corrections in the limit we are considering.

On the other hand, the requirement of $\phi$ to be single valued implies that $\alpha(2 \pi)=2 \pi n$, with $n$ an integer. A suitable (smooth) gauge transformation allows one to choose $\alpha=n \varphi$. Then, we conclude that the magnetic flux is quantized,

$$
\begin{aligned}
\Phi_{\theta} & =\int d^{2} x B_{\theta}=\oint_{r \rightarrow \infty} d \vec{l} \cdot \vec{A}=\frac{1}{e}[\alpha(2 \pi)-\alpha(0)] \\
& =\frac{2 \pi}{e} n .
\end{aligned}
$$

But, as we will see later, the charge density is not proportional to the flux as in the commutative case.

To solve the equations of motion, we adopt the usual ansatz [7]

$$
\begin{aligned}
& A_{\varphi}=\frac{\sqrt{2 \tilde{\phi}_{0}^{*} \tilde{\phi}_{0}}}{u}(n-g(u)), \\
& A_{0}=\sqrt{2 \tilde{\phi}_{0}^{*} \tilde{\phi}_{0}} h(u), \\
& A_{3}=A_{r}=0, \quad \phi=\sqrt{\tilde{\phi}_{0}^{*} \tilde{\phi}_{0}} f(u) e^{i n \varphi},
\end{aligned}
$$

where $u=r \sqrt{2 e^{2} \tilde{\phi}_{0}^{*} \tilde{\phi}_{0}}$.

Equations of motion (11) now reduce to

$$
\begin{aligned}
h^{\prime \prime}+\frac{1}{u} h^{\prime}-f^{2} h & =\frac{\tilde{\theta}}{u} g^{\prime} \\
g^{\prime \prime}-\frac{1}{u} g^{\prime}-f^{2} g & =\tilde{\theta} u h^{\prime} \\
f^{\prime \prime}+\frac{1}{u} f^{\prime}-\frac{g^{2}}{u^{2}} f-\frac{\lambda}{4 e^{2}}\left(f^{2}-1\right) f & =-h^{2} f,
\end{aligned}
$$

where the primes stand for derivatives with respect to $u$ and $\tilde{\theta}=\theta / \sqrt{2 e^{2} \tilde{\phi}_{0}^{*} \tilde{\phi}_{0}}$.

Equations (16a)-(16c) coincide with those found in Ref. [5] in the context of charged vortex solutions. The energy per unit of length for this solution, given by

$$
\begin{aligned}
\mathcal{E}_{n}= & 2 \pi \tilde{\phi}_{0}^{*} \tilde{\phi}_{0} \int_{0}^{\infty} u d u\left\{\frac{1}{u^{2}}\left(\frac{d g}{d u}\right)^{2}+\frac{1}{2}\left(\frac{d f}{d u}\right)^{2}+\left(\frac{d h}{d u}\right)^{2}\right. \\
& \left.+\frac{1}{2}\left[\left(\frac{g}{u}\right)^{2}+h^{2}\right] f^{2}+\frac{\lambda}{16 e^{2}}\left(f^{2}(u)-1\right)^{2}\right\},
\end{aligned}
$$

turn out to be finite, up to an additive constant, for a suitable class of boundary conditions.

Remarkably, the modified potential (10) allows a direct analysis of the conditions under which vortex solutions exist in the presence of this kind of noncommutativity. Indeed, we can rewrite (10) as

$$
\tilde{V}(\rho)=\frac{\lambda}{4}\left[\rho-\left(\rho_{0}-\frac{\Theta^{2}}{2 \lambda}\right)\right]^{2}+\frac{\Theta^{2}}{4}\left(\rho_{0}-\frac{\Theta^{2}}{4 \lambda}\right),
$$

where the last term is a physically irrelevant constant and the first one can be written as $V\left(\rho-\tilde{\rho}_{0}\right)$. Clearly, if $\tilde{\rho}_{0}:=$ $\rho_{0}-\frac{\Theta^{2}}{2 \lambda} \leq 0$ then the system does not present the symmetry breaking phenomenon and it is not possible to find vortexlike solutions. Furthermore, for $\tilde{\rho}_{0}=0$ one can see that the energy is zero.

Hence, one can expect that, in order to find finite linear energy density vortexlike solutions, the condition $\phi_{0}^{*} \phi_{0}>$ $\frac{\Theta^{2}}{2 \lambda}$ should be satisfied.

Concerning the linear charge density, given in (12), we get from (15)

$$
Q_{\theta}=\frac{2 \pi \theta}{e} n-\frac{\pi \Theta}{e} \int d u u f^{2}(u) .
$$

The last term is independent of $\tilde{\phi}_{0}$ and, since we know from [5] that, for large $u$,

$$
f(u) \rightarrow 1-e^{-a u}, \quad \text { with } a=\frac{\sqrt{(\theta / 2)^{2}-e^{2} \tilde{\rho}_{0}}-(\theta / 2)}{\sqrt{2 e^{2} \tilde{\rho}_{0}}},
$$

we can argue that the divergent part of (19) is a contribution from the nontrivial vacuum with no physical consequences. Indeed, the difference $\Delta_{n m} Q:=Q_{n}-Q_{m}$ is finite (notice that the function $f$ does not depend on $n$ ) for any $n$ and $m$, including $n$ or $m$ equal to zero.

\section{FINAL REMARKS AND CONCLUSION}

Let us summarize and comment our results. We have found that there exist charged vortex solutions in noncommutative scalar electrodynamics which have similar properties to those found in [5] for the commutative case. But in the present case, these solutions depend on the noncommutativity parameters that deform the scalar and vectorial momenta commutators.

As remarkable differences with respect to the commutative case, we can point out that the charge of these vortices has not a topological character and that, even for a nonzero vacuum value of the Higgs fields, there are no vortex solutions except that $\left|\phi_{0}\right|^{2}$ be larger than a bound proportional to $\Theta^{2}$, where $\Theta$ is the noncommutativity parameter of the Higgs field.

The point of view advocated in this paper is intimating related to the Kostelecký et al. approach [15], however a more precise connection and, in particular, the possible 
connection with the vortex solutions found here is under research.

\section{ACKNOWLEDGMENTS}

This work was supported from FONDECYT Grants No. 1050114 (J.G.), 1060079 (F.M.), and 3060002
(J.L.-S.). The work of A. J.S. was supported in part by FAPESP (Fundacão de Amparo a Pesquisa do Estado de São Paulo) and CNPq (Conselho Nacional de Desenvolvimento Científico e Tecnológico). H.F. acknowledges support from CONICET (PIP 6160) and UNLP (Proy. 11/X381), Argentina.
[1] K. Shinozaki et al. (AGASA Collaboration), Nucl. Phys. B, Proc. Suppl. 136, 18 (2004); M. Takeda et al., in Hamburg 2001, Cosmic Ray (ICRC, Hamburg, 2001), p. 341.

[2] C. Athanassopoulos et al. (LSND Collaboration), Phys. Rev. Lett. 81, 1774 (1998).

[3] M. Bridges et al., astro-ph/0607404.

[4] For a review on vortex solutions, see e.g., E. D'Hoker and L. Vinet, Ann. Phys. (N.Y.) 162, 413 (1985).

[5] A. Khare, Proc. Indian Natl. Sci. Acad. A 61, 161 (1995).

[6] A. A. Abrikosov, Sov. Phys. JETP 5, 1174 (1957).

[7] H. B. Nielsen and P. Olesen, Nucl. Phys. B61, 45 (1973).

[8] T. W. B. Kibble, Phys. Rep. 67, 183 (1980).

[9] E. Witten, Phys. Lett. 153B, 243 (1985); J. Polchinski, hep-th/0412244; J. D. Edelstein, C. Núñez, and F. A. Schaposnik, Phys. Lett. B 329, 39 (1994); Nucl. Phys. B458, 165 (1996); Phys. Lett. B 375, 163 (1996); M. Shifman and A. Yung, Phys. Rev. D 70, 025013 (2004); G. Dvali, R. Kallosh, and A. Van Proeyen, J. High Energy Phys. 01 (2004) 035; P. Binetruy, G. Dvali, R. Kallosh, and A. Van Proeyen, Classical Quantum Gravity 21, 3137 (2004); J. López-Sarrión, E. Moreno, F. A. Schaposnik, and D. Slobinsky, Phys. Rev. D 73, 125007 (2006).

[10] J. Gamboa and J. López-Sarrión, Phys. Rev. D 71, 067702 (2005).

[11] A. Das, J. Gamboa, J. López-Sarrión, and F. A. Schaposnik, Phys. Rev. D 72, 107702 (2005).

[12] H. Falomir, J. Gamboa, J. López-Sarrión, F. Mendez, and
A. J. da Silva, Phys. Lett. B 632, 740 (2006); J. Gamboa, J. López-Sarrión, and A. P. Polychronakos, Phys. Lett. B 634, 471 (2006).

[13] J. M. Carmona, J. L. Cortés, J. Gamboa, and F. Mendez, J. High Energy Phys. 03 (2003) 058; Phys. Lett. B 565, 222 (2003).

[14] S. Carroll, G. Field, and R. Jackiw, Phys. Rev. D 41, 1231 (1990); A. A. Andrianov, P. Giacconi, and R. Soldati, J. High Energy Phys. 02 (2002) 030.

[15] D. Colladay and V. A. Kostelecký, Phys. Lett. B 511, 209 (2001); V. A. Kostelecký and R. Lehnert, Phys. Rev. D 63, 065008 (2001); R. Bluhm and V. A. Kostelecký, Phys. Rev. Lett. 84, 1381 (2000); V. A. Kostelecký and C. D. Lane, Phys. Rev. D 60, 116010 (1999); R. Jackiw and V. A. Kostelecký, Phys. Rev. Lett. 82, 3572 (1999); D. Colladay and V. A. Kostelecký, Phys. Rev. D 58, 116002 (1998); V. A. Kostelecký and R. Potting, Phys. Lett. B 381, 89 (1996); R. Potting and R. Lehnert, Phys. Rev. Lett. 93, 110402 (2004); O. Bertolami, D. Colladay, V. A. Kostelecký, and R. Potting, Phys. Lett. B 395, 178 (1997); S. A. Paul and A. Khare, Phys. Lett. B 174, 420 (1986); 177, 453(E) (1986).

[16] D. P. Jatkar, G. Mandal, and S. R. Wadia, J. High Energy Phys. 09 (2000) 018.

[17] G. Lozano, M. V. Manias, and F. A. Schaposnik, Phys. Rev. D 38, 601 (1988); Zheng-Min Xi, Phys. Lett. 217, 113 (1988); Choonkyu Lee, Kimyeong Lee, and Hyunsoo Min, Phys. Lett. 252, 79 (1990). 